\title{
ON NONLINEAR DESINGULARIZATION
}

\author{
BY M. S. BERGER ${ }^{1}$ AND L. E. FRAENKEL
}

"Nonlinear desingularization" is an interesting, hitherto little studied phenomenon in nonlinear elliptic partial differential equations. By nonlinear desingularization we mean that a linear boundary value problem whose solution possesses one or more isolated singularities is a degenerate form of a family of nonlinear problems whose solutions are smooth, and moreover converge to the singular solution of the linear system upon degeneration. Such phenomena occur commonly in various branches of theoretical physics. For example, in Helmholtz's famous study of vortex motion of ideal fluids [1], a circular vortex filament is used to approximate a steady vortex ring of small cross section. In that case the Stokes stream function of the vortex filament is the Green's function for the axisymmetric Laplace operator, and hence has a singularity, while the Stokes stream function for the vortex ring of small cross-section satisfies a nonlinear elliptic partial differential equation, and is smooth [2]. Similar phenomena occur in the plasma physics of Tokomaks governed by the Lundquist equations, and in the onset of the "mixed state" [3] in type II superconductors associated with the Ginzberg-Landau equations. Moreover in [4], S. Adler has discussed how the nonlinear desingularization process leads to new classes of static Euclidean $S U(2)$ Yang-Mills fields (monopoles) with unit Pontrajagin index.

Mathematically speaking, nonlinear desingularization is a rather novel kind of bifurcation phenomenon involving parameter-dependent nonlinear problems. Indeed, we shall indicate in $\$ 3$ below how our ideas on bifurcation, contained in [5], have an exact parallel in a typical situation of nonlinear desingularization.

1. The nonlinear problem and its linear degenerate form. Let $\Omega$ be a bounded domain in the plane $\mathbf{R}^{2}$, with boundary $\partial \Omega$, and let $L$ be a formally self-adjoint, uniformly elliptic, second order operator, with smooth coefficients $a_{i j}(x)$ defined on $\Omega$ with the matrix $\left(a_{i j}(x)\right)$ positive definite and

$$
L u=-\sum_{i, j=1}^{2} \frac{\partial}{\partial x_{1}}\left(a_{i j}(x) \frac{\partial u}{\partial x_{j}}\right) .
$$

Then we consider the free boundary problem of finding a piecewise smooth

Received by the editors July 23, 1979 and, in revised form, August 14, 1979. AMS (MOS) subject classifications (1970). Primary 39A15; Secondary 47D20. ${ }^{1}$ Research partially supported by NSF and AFOSR grants. 
function $u$ and a connected subdomain $A$ of $\Omega$ such that

$$
\begin{aligned}
L u & =\left\{\begin{array}{cl}
\lambda f(x, u) & \text { on } A, \\
0 & \text { on } \Omega-\bar{A},
\end{array}\right. \\
\left.u\right|_{\partial A} & =0 \text { and }\left.u\right|_{\partial \Omega}=-q_{0}(x, R), \quad u(x) \text { is } C^{1} \text { across } \partial A .
\end{aligned}
$$

Here $q_{0}(x, R)$ is a given positive smooth function defined on $\partial \Omega \times \mathbf{R}^{+}$. The $C^{1}$ function $f(x, t)$ defined on $\Omega \times \mathbf{R}^{+}$is nonnegative, convex in $t$ for fixed $x$ and $t \geqslant 0$, and vanishes identically for $t \leqslant 0$. Moreover $f(x, t)$ satisfies a polynomial growth condition as $t \rightarrow \infty$, while as $t \rightarrow 0, f(x, t) \geqslant M t^{m}$ for some fixed absolute positive constants $M$ and $m$, with $m>1$. The numbers $\lambda$ and $R$ are positive parameters to be specified below. The smooth function $q_{0}(x, R)$ is uniformly bounded away from zero as the parameter $R$ varies, but is allowed to tend to infinity at $R \rightarrow 0$. The special case $f(x, t)=g(x) t$ can also be included.

A linear limiting special case of this problem is obtained by supposing that $\bar{A}$ shrinks to a point $a \in \Omega$ in such a way that $\lambda \int_{\Omega} f(x, u) d x$ remains finite, and tends to a limiting value $c$ (say). Then the linear degenerate form of (1) would be

$$
L u=c \delta(x-a),\left.\quad u\right|_{\partial \Omega}=-q_{0}(x, R)
$$

where $\delta(x-a)$ denotes the appropriate Dirac delta function. Thus the solution of (2) is the difference of two terms, one smooth term $q(x, R)$ representing the $L$-harmonic extension of $q_{0}(x, R)$ into $\Omega$, and another which is proportional to the Green's function $G(x, a)$ of $L$ relative to $\Omega$.

It is convenient then to reformulate (1) in terms of the function $U=u+$ $q$. Indeed (1) becomes

$$
L U=\lambda f(x, U-q), \quad U \mathrm{I}_{\partial \Omega}=0 .
$$

The free boundary $\partial A$, then becomes the set of points in $\Omega$ where $U=q$ and the set $A=\{x \in \Omega \mid U>q\}$.

2. The nonlinear desingularization theorem. To demonstrate the nonlinear desingularization phenomenon for (1) it is necessary to show that the Green's function solution of (2) referred to above is the degenerate limit of a family of smooth solutions to the full free boundary problem (1), by letting the parameters $\lambda$ and $R$ vary. This fact is the content of the following result.

THEOREM 1. Under the above hypotheses, there is a one parameter family of solutions $\left(u_{R}, \lambda_{R}\right)$ with $u_{R} \in C^{1}(\bar{\Omega})$ and connected subdomains $A_{R}$ of $\Omega$ satisfying (1), as the parameter $R$ varies over the positive real numbers. This family and the parameter $R$ are characterized by the isoperimetric variational problem defined in (4) below. Moreover as $R \rightarrow 0$, (i) $\lambda_{R} \rightarrow \infty$, (ii) the diameter of $A_{R}$ tends to 0 , and (iii) setting $h_{R}=\lambda_{R} \int_{A_{R}} f\left(x, u_{R}\right) d x$ and 
$u_{R}=U_{R}-q(x, R)$

$$
\left\|U_{R} / h_{R}-G(\cdot, a(R))\right\|_{W_{1, P}(\Omega)} \rightarrow 0
$$

where $1 \leqslant p<2$, and $a(R)$ is any point in $A_{R}$.

REMARK. The statement (3) is sharp, in the sense that it is false if $p=2$.

3. On the proof of Theorem 1. The proof utilizes the method of "nonlinear descent" introduced in [5] for bifurcation problems. Instead of considering (1) as a nonlinear perturbation of (2), one uses global nonlinear methods to solve (1) and then considers the behaviour of the solutions of the nonlinear problems under the appropriate limiting operations. To solve the nonlinear problem (1) in this case we consider the isoperimetric variational problems

$$
\left[V_{R}\right]: \inf _{U \in \Sigma_{R}} D[U]
$$

where $\Sigma_{R}=\left\{U \mid U \in \stackrel{\circ}{W}_{1,2}(\Omega), \int_{\Omega} F(x, U-q)=R\right\}$ with $f(x, t)=F_{t}(x, t)$ and $F(x, 0)=0$, and $D[U]$ is the Dirichlet form associated with the operator $L$. Using the arguments of [2], we find that the critical points of this variational problem generate the desired one parameter family of solutions $\left(u_{R}, \lambda_{R}\right)$ for the free boundary problem (1). We then consider the behaviour of the solutions of $\left[V_{R}\right]$ as $R \rightarrow 0$. To this end we show that as $R \rightarrow 0$, the parameter $\lambda_{R}$ in (1) tends to $+\infty$. Moreover, setting

$$
A_{R}=\left\{x \mid x \in \Omega, U_{R}>q(x, R)\right\}
$$

and cap $\left(\bar{A}_{R}, \Omega\right)=\inf _{T_{R}} D[u]$, where $T_{R}=\left\{u \in \stackrel{\circ}{W}_{1,2}(\Omega), u \geqslant 1\right.$ on $\left.\bar{A}_{R}\right\}$, we show $\operatorname{cap}\left(\bar{A}_{R}, \Omega\right) \rightarrow 0$ as $R \rightarrow 0$. This implies that the diameter of $A_{R}$ tends to zero at a known rate [6], since $\Omega$ is a plane domain. The limit (3) then follows from Minkowski's inequality applied to an integral equation satisfied by $\left(U_{R}, \lambda_{R}\right)$.

\section{BIBLIOGRAPHY}

1. H. Helmholtz, On integrals of the hydrodynamical equations which express vortex motion, Crelle's J. 55 (1858), 25-55.

2. L. E. Fraenkel and M. S. Berger, $A$ global theory of steady vortex rings in an ideal fluid, Acta Math. 32 (1974), 13-51.

3. A. Abrikosov, Soviet Physics JETP, 5, 1957, pp. 1174-1192.

4. S. Adler, Global structure of static SU(2) solutions, Physical Rev. D 20 (1979), $1386-1411$.

5. M. S. Berger, $A$ bifurcation theory for nonlinear elliptic partial differential equations. Bifurcation Theory (J. Keller and S. Antman), Benjamin, New York, 1969, pp. 113216.

6. L. E. Fraenkel, On Steiner symmetrization and a lower bound for electrostatic capacity in the plane (to appear).

DEPARTMENT OF MATHEMATICS AND STATISTICS, UNIVERSITY OF MASSACHUSETTS, AMHERST, MASSACHUSETTS 01003

MATHEMATICS DIVISION, UNIVERSITY OF SUSSEX, BRIGHTON, BN1 9QH, 